www.nature.com/clinicalpractice/cardio

Although fosinopril doses used in this trial were extrapolated from adult data, no dose-response relationship was seen and there was unexpected sensitivity to lower doses of the drug. The authors recommend that starting doses for children should be $\leq 0.1 \mathrm{mg} / \mathrm{kg}$.

Original article Li JS et al. (2004) Is the extrapolated adult dose of fosinopril safe and effective in treating hypertensive children. Hypertension 44: 289-293

\section{Early, aggressive statin therapy in ACS}

Results from phase $Z$ of the international $A$ to $Z$ trial have now been reported. This part of the study compared early, aggressive statin therapy in Acs with the standard approach of a delayed, less-intensive regimen.

Patients with ACS were randomized to $40 \mathrm{mg} /$ day simvastatin for 1 month and $80 \mathrm{mg} /$ day thereafter $(n=2,265)$, or placebo for 4 months followed by $20 \mathrm{mg} /$ day simvastatin $(n=2,232)$. Follow-up was for $6-24$ months and the primary efficacy endpoint was a composite of cardiovascular death, nonfatal MI, readmission for ACS and stroke.

The trial did not achieve the prespecified endpoint, partly due to a lower than expected number of endpoint events and a high (33\%) rate of study-drug discontinuation. LDL cholesterol levels were significantly lower in the simvastatin-only group patients than in those taking placebo plus simvastatin. Despite this difference, there was no statistically significant difference in the primary endpoint rate between the two groups, although there was a trend toward a reduction in the simvastatinonly group. Between 4 and 24 months, however, the primary endpoint was significantly lower in the simvastatin only group (HR 0.75, $95 \% \mathrm{Cl} 0.60$ to $0.95, P=0.02$.).

Concluding that an overall trend toward reduction of major cardiovascular events had been shown in the simvastatin-only group, the authors propose that statins should be started early after ACS, at doses higher than the typical starting dose. They caution that myopathy was more common among patients receiving $80 \mathrm{mg} /$ day of simvastatin than those receiving lower doses of the drug.

Original article De Lemos JA et al. (2004) Early intensive vs a delayed conservative simvastatin strategy in patients with acute coronary syndromes. JAMA 292: 1307-1316

\section{Proapoptotic serum activity and mortality in HF}

Serum of patients with heart failure (HF) can induce apoptosis in cultured endothelial cells. This observation prompted Rössig and colleagues to consider whether proapoptotic activity is related to disease progression.

Serum samples were obtained from 48 patients with HF and systemic proapoptotic activity was determined using an ex vivo cell-culture assay. Patients were then followed up for a minimum of 30 months, with all-cause mortality being the single endpoint of the study.

A total of 16 patients died during follow-up; 11 received a heart transplant and were considered as survivors. Mortality was significantly higher in patients whose serum proapoptotic activity was above the median value $(P<0.02)$. Multivariate regression analysis revealed that proapoptotic serum activity was an independent predictor of death (HR 1.85 per \%, $P=0.008$ ), as were elevated PRO-BNP levels (HR 9.35 per $\log [$ pro-BNP], $P=0.001$ ) and low blood pressure (HR 0.96 per $\mathrm{mmHg}, P=0.041$ ). The sensitivity and specificity of serum pro-apoptotic activity analysis for predicting mortality were similar to those for pro-BNP serum levels.

Concluding that proapoptotic serum activity is an independent predictor of HF mortality, the authors suggest that it may provide a measure of the effects of systemic inflammation in these patients.

Original article Rössig L et al. (2004) The pro-apoptotic serum activity is an independent mortality predictor of patients with heart failure. Eur Heart J 25: 1620-1625

\author{
GLOSSARY \\ ACS \\ Acute coronary syndromes \\ MI \\ Myocardial infarction \\ PRO-BNP \\ NT-pro-brain natriuretic \\ peptide \\ HR \\ Hazard ratio
}

